\title{
Real-time study of remote highway snow warning technology Zhao Huaqi ${ }^{1, a}$, Xiang Changyong,Li Guojing ${ }^{2, b} *$ (Author for correspondence), Wang Bin, Zhang Shijie, Wen Dazhi \\ ${ }^{1}$ College of Information Science and Electronic Technology, Jia Musi University, Hei Longjiang Province Jia Musi 154007, China \\ ${ }^{2}$ School of Materials Science and Engineering, Jia Musi University, Hei Longjiang Province Jia Musi 154007, China \\ ${ }^{12}$ zhaohuaqi@jmsu.edu.cn \\ ${ }^{2 b}$ li_guojing@126.com
}

Keywords: single chip microcomputer; SIM900A; GSM; Paragraphs road snow thickness; warning

\begin{abstract}
Snowfall directly affects the traffic safety and a lot of accidents happened every year because of the snowfall. If the traffic department can timely detect the thickness of the snow on the highway and clear the snow in time, the rate of traffic accident will reduce and won' $t$ make much trouble for people. Therefore, the research on remote real-time monitoring of highway snow will solve this problem. This device uses single chip microcomputer of STC12 series as the controller, SIM900A as communication module and will sent the data of the snow thickness monitored remotely to the traffic departments, which makes that can get real-time online warned. Using GSM communication system, snow forecast and alarm can be realized in areas covered by mobile network and it is not affected by outside conditions such as weather and terrain, which is an ideal device for early warning communication system.
\end{abstract}

\section{Introduction}

With the development of the national economy, the national science and technology are developing, technology of wireless communication is becoming more and more mature, and the application of radio communication technology field is also very wide. However, most of the application in the road is the radar speed measuring and monitoring, snow conditions for pavement snow thickness detection warning is seldom study [1]. But the great influence the thickness of snow in the pavement snow day case on transportation, is one of the important factors causing traffic accidents. When the traffic department encountered snow weather in normal circumstances, in order to avoid the occurrence of traffic accidents, they basically took highway blocking measure and banned the vehicle traffic, which is not the best way to solve this problem and makes people travel inconvenient.

Therefore, research on remote real-time monitoring of highway snow can solve this problem. Through the installation of the device in real time field analysis of snowfall conditions in each section of the road, when a section of highway due to snowfall impact the normal operation of highway traffic, the device will send a message to remind the good road snow cleaning work. The highway department staff on the device for human-computer interaction based on field environment the off highway set the corresponding threshold on the device. If bad weather continued to drop heavy snow, prompting the local highway section staff blockade highway. When the staff receive the information and carry out the relevant work, they can send "OK" to the device, the device will no longer send a message. So that it can reduce the staff's highway workload and does not affect people's travel. The system realizes the function of interactive remote real time early warning of highway snow condition. 


\section{The realization of system functions}

The road test of SIM900A forecast snow field monitoring and alarm system is shown in Figure 1. The device consists of highway snow condition monitoring module, MCU, SIM900A module, GSM signal of the base station and mobile phone user terminal. The road snow module is to use ultrasonic monitoring, and use single-chip to receive data collected by highway snow condition monitoring module, and make the appropriate analysis of the data, and then the MCU uses AT commands to control SIM900A module and through the GSM signal transmits to the terminal road work personnel corresponding data, forecast and alarm information. So the relevant staff can receive can receive snowfall conditions of highway and related data as long as the GSM signals cover the area [2,3]. Since all the workers on this road previously stored the mobile phone card number in the SIM900A module, when the device sends information to the terminal staff, it can send information simultaneously to multiple users, so that staff do not have to tell each other and inform, and can directly do their own work. After the reception of information, staff return "OK" to the device and then device will no longer send information. Realization of remote wireless man-machine interaction, it can make that each highway section staff do not have to travel to the actual site to survey the actual pavement snow conditions, they can know the snowfall conditions on the road.

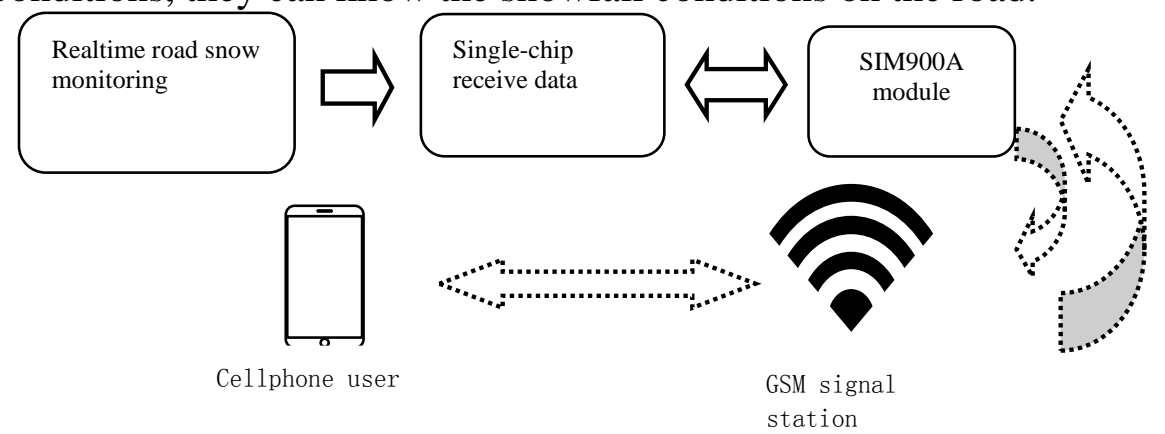

Figure 1.The road test of SIM900A forecast snow field monitoring and alarm system is shown

\section{System analysis}

\subsection{Hardware facilities}

The hardware connection of MCU and SIM900A module is diaplayed in diagram of Figure 2. Connecting one of the microcontroller and SIM900A module requires only three wires to realize communication, at the same time, in order to avoid communication error occurred two modules share a power supply.

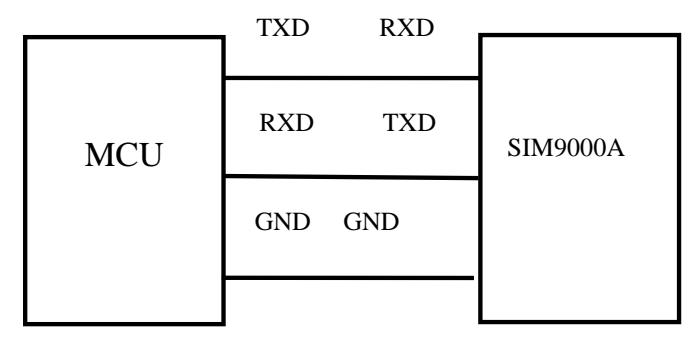

Figure 2.MCU and SIM900A module of the hardware connection diagram

Here monolithic computer model is STC12C5A60S2, which is a new generation of 8051 single chip, which has the features of high speed, low power consumption, super anti-interference. Flash memory capacity is only 60K. This single-chip microcontroller development requires simple equipment, so the programmer time is greatly shortened in the development [4]. Encryption can be 
used in the program within the microcontroller, thus, it can well protect the fruits of their labour. The smallest single chip system is as shown in figure 3.

SIM900A is a GSM/GPRS module designed by the SIMCOMcompany especially for the China market. The working frequency is EGSM $900 \mathrm{MHz}$ and DCS $1800 \mathrm{MHz}$. It supports GPRS multi-slot class 10/class 8 and GPRS code format CS-1, CS-2, CS-3 and CS-4. SIM900A interior is embedded TCP/IP protocol, which is very practical for data transmission [5].

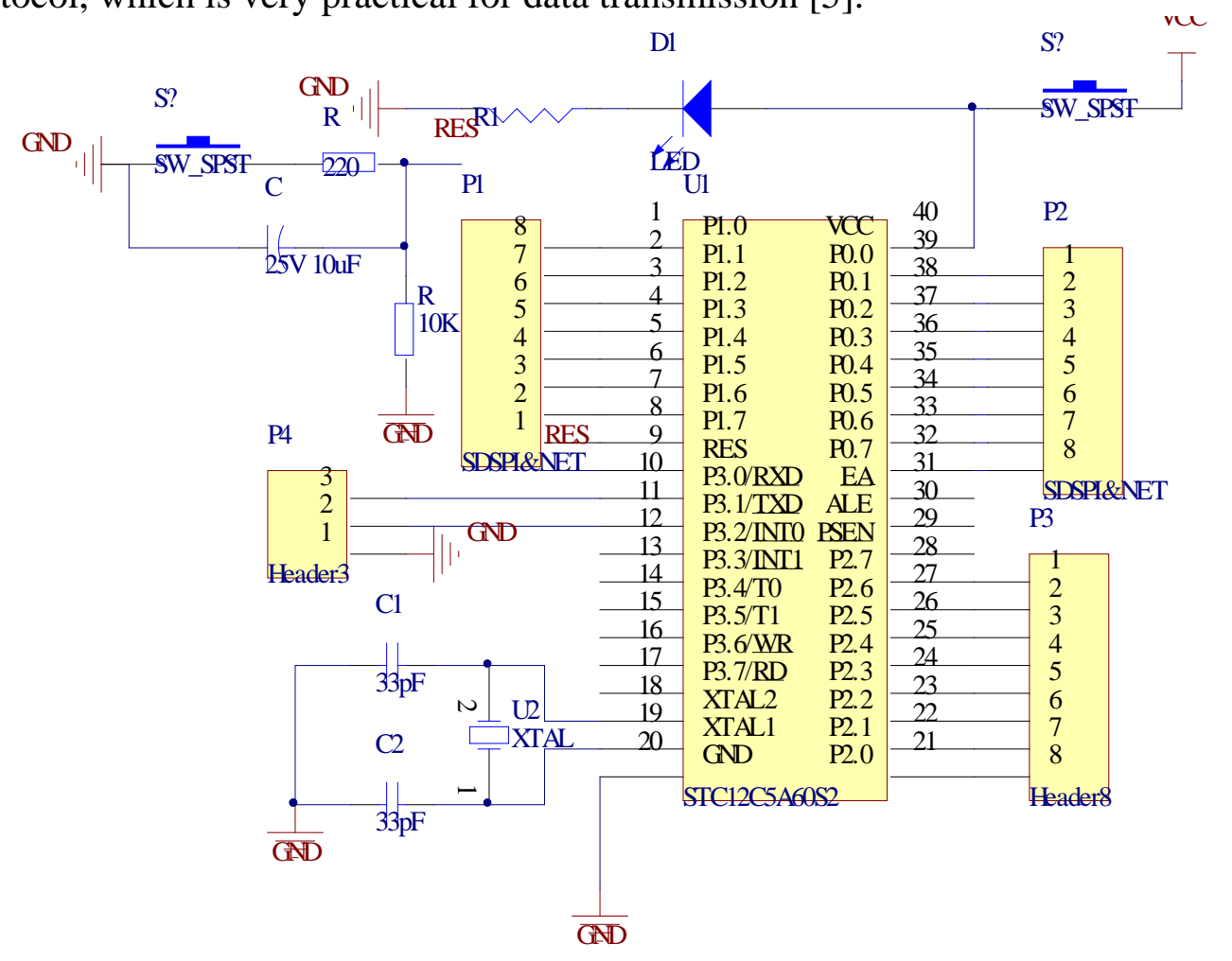

Figure 3.The smallest single chip system as shown

\section{2 software design}

The command sent by microcontroller to the SIM900A must be sent as a ASCII encoded character format, and instruction cannot appear in Chinese. There are some difficulties here, the device SIM900A use USC2 coding method for text editing and analyzing. When we use SCM to edit text contents and read the message contents to the SIM900A, we need Chinese, digital and UNISODE character conversion. Because the number of characters applied in the forecast and alarm device is not too much, so we can directly establish a UNISODE character database in single-chip microcomputer, it can be used [6-9].

Here, for the use of single-chip machine language is the $C$ language, we began to use a switch statement (switch) to determine whether SCM receives snowfall data monitoring device, if it is the execution of a program on the SIM900A module of AT commands to send to the terminal highway department staff to send "*** when a fraction of a day section of highway snow". Then the MCU to analyze the received data, because the road test device using ultrasonic measurement of changes in snow is snow depth to determine the snowfall conditions, therefore, data analysis is to change the pavement thickness of snow's analysis of the situation. The specific algorithm:

The snowfall rate $A=$ Changes in surface snow depth $\triangle B$ /Time variation $\triangle T \quad$ ( 1 )

We judge A variable changes. If the A changes in the threshold of 1 description data in the normal changes at a given time, the device will send to send a message "* day * time, on a section of highway pavement thickness of snow is a few millimeters, and it continues to snow (or snow has stopped)" to the terminal road staff; if the change of variable $A$ at a threshold of 2 indicates that the road condition within this field deteriorated, the editor of SIM900A forecasts and sends information "* day * time, on a section of highway pavement thickness of snow is a few millimeters, pavement thickness of 
snow has reached a snow range, please do the snow cleaning work as soon as possible, so as to avoid effect on highway traffic. And it continues snowfall ( or it has stopped)" to the terminal road staff. When the variable A to maximum threshold of 3 , the device will send alarm information to the terminal from time to time, and SIM900A will edit forecast information and send a message "* day * time, on a section of highway pavement thickness of snow has been more than a few millimeters, and thickness of snow has been out of automotive safety driving range, please blockade the road as soon as possible and vehicles traffic are not allowed, so as to avoid effects on road traffic safety. It continues to snow (or it has stopped )" to the terminal road staff. If the user receives the warning information and sends back OK to the system, the system will no longer send alarm information.

SCM and SIM900A communication used serial mode 1, and it must be guaranteed that the communication baud rate of two modules are exactly the same. when receiving data and sending data we should pay attention to that SCM must firstly put the serial port interrupted and closed in transmitting data, otherwise it will also enter the serial port and interrupt service routine, thus it will affect the normal running of the program. General microcomputer software flow chart is shown in figure 4.

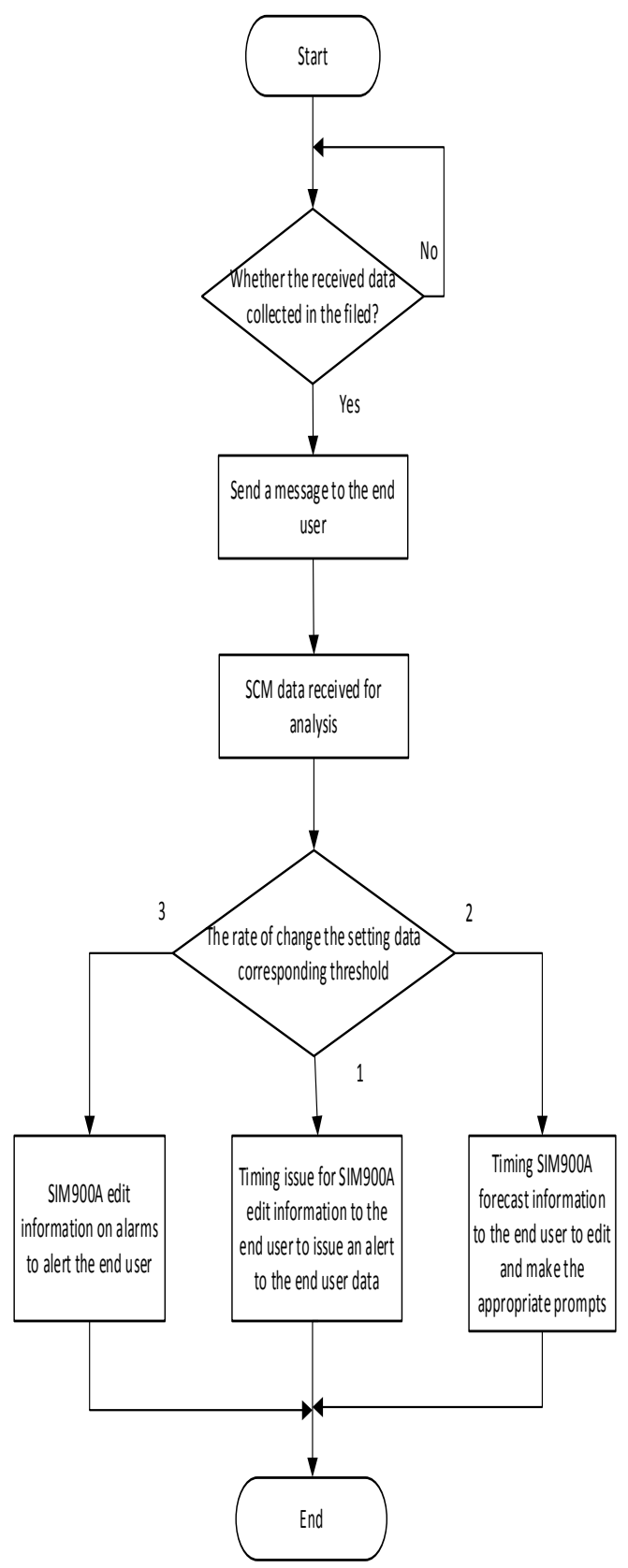

Figure 4.General microcomputer software flow chart in figure 


\section{Summary}

The research on remote real time early warning technology of highway snow takes MCU and SIM900A module as the core, and has the function of notifying staff real-time snowfall conditions of highway and allowing them to take emergency measures. It brings great convenience to the highway related staff, also reduces traffic accidents, and at the same time it improves security for people travel. The device has been tested in the actual practice, and it has strong stability.

\section{Acknowledgment}

This research was supported by the National Natural Science Fund( Grant No.51278227), the National Science Foundation of Heilongjiang Province (Grant No. E201212), 2014 college students innovation and entrepreneurship training programs of Heilongjiang province( Grant No. 201410222022), The key research project of Jia Musi 2011 Annual(Grant No. 11074), Fund of talent cultivation of Jiamusi University(Grant No. RC2010-027), Project of Science and technology of Jiamusi University (Grant No. L2012-003), the National Science Foundation of Jiamusi University (Grant No. Lz2014-005), all support is gratefully acknowledged.

\section{References}

[1] Zhang Xuetong Qinghai province snow detection and snow disaster in pastoral area warning research [D]. Lanzhou: Lanzhou University, 2010

[2] Cao Weiqin, Han Bing. Realization of remote monitoring and control of [J]. using GSM Short.Message radio engineering, 2002,32 (10): 21-23.

[3] Ma Chao. Embedded GSM short message interface hardware and software design of [J]. microcontroller and embedded systems should be used, 2003 (8): 15-17.

[4] Guo tianxiang. The first concept of 51 single-chip C language tutorial -- into the door, increase, development, expansion of Raiders [M]. Beijing: Electronic Industry Press, 2009

[5] Di Shun, Wang WeiHong, Li Pen. The Internet of things based on SIM900A SMS alarm system [J]. modern electronic technology,.2012,35 (5): 86-8.

[6] Qiao Guopen STM32 and AM1705 design of the [D]. vehicle real-time monitoring system of Chengdu University of Technology based on.2012.5.

[7] Li Yongsheng, Huang Lanhong, Liu Hongjun. Multi file UDP protocol transmission [J]. Journal of Guangxi University For Nationalities: Natural Science Edition. Based on 2007,13 (2): 68-71.

[8] Wang Zhu, Hao Xiaoqiang, Wei Debao. WSN and GPRS network of remote water quality monitoring system [J]. instrument technique and sensor based on.2010 (1): 48-52.

[9] Wang Zhu, Hao Xiaoqiang, Wei Debao. WSN and GPRS network of remote water quality monitoring system [J]. instrument technique and sensor based on.2010 (1): 48-52. 